\title{
Dissociable Contributions of the Orbitofrontal and Infralimbic Cortex to Pavlovian Autoshaping and Discrimination Reversal Learning: Further Evidence for the Functional Heterogeneity of the Rodent Frontal Cortex
}

\author{
Y. Chudasama and Trevor W. Robbins \\ Department of Experimental Psychology, University of Cambridge, Cambridge, United Kingdom CB2 3EB
}

\begin{abstract}
To examine possible heterogeneity of function within the ventral regions of the rodent frontal cortex, the present study compared the effects of excitotoxic lesions of the orbitofrontal cortex (OFC) and the infralimbic cortex (ILC) on pavlovian autoshaping and discrimination reversal learning. During the pavlovian autoshaping task, in which rats learn to approach a stimulus predictive of reward [conditional stimulus $\left(\mathrm{CS}^{+}\right)$], only the OFC group failed to acquire discriminated approach but was unimpaired when preoperatively trained. In the visual discrimination learning and reversal task, rats were initially required to discriminate a stimulus positively associated with reward. There was no effect of either OFC or ILC lesions on discrimination learning. When the stimulus-reward contingencies were reversed, both groups of animals committed more errors, but only the OFC-lesioned animals were unable to suppress the previously rewarded stimulus-reward association, committing more "stimulus perseverative" errors. In contrast, the ILC group showed a pattern of errors that was more attributable to "learning" than perseveration. These findings suggest two types of dissociation between the effects of OFC and ILC lesions: (1) OFC lesions impaired the learning processes implicated in pavlovian autoshaping but not instrumental simultaneous discrimination learning, whereas ILC lesions were unimpaired at autoshaping and their reversal learning deficit did not reflect perseveration, and (2) OFC lesions induced perseverative responding in reversal learning but did not disinhibit responses to pavlovian $\mathrm{CS}^{-}$. In contrast, the ILC lesion had no effect on response inhibitory control in either of these settings. The findings are discussed in the context of dissociable executive functions in ventral sectors of the rat prefrontal cortex.
\end{abstract}

Key words: rat; autoshaping; orbitofrontal cortex; infralimbic cortex; inhibition; perseveration; reversal learning; discrimination

\section{Introduction}

Executive functions of the prefrontal cortex (PFC) include the capacity for responding to changing environmental contingencies (Miller, 2000). Neurophysiological (Tremblay and Schultz, 1999) and functional neuroimaging (O’Doherty et al., 2001) data show important roles for the PFC in the representation of reward. Moreover, lesion studies (Butter, 1969; Iversen and Mishkin, 1970; Dias et al., 1996) and electrophysiological observations in monkeys (Miller, 2000; Rolls, 2000) implicate certain regions of the PFC in associative learning. Additionally, deficits in reversal learning tasks (Rolls et al., 1994; Rahman et al., 1999) and acquiring contingencies in complex decision-making tasks (Bechara et al., 1994) in human patients have implicated the orbitofrontal cortex (OFC) in stimulus-reinforcer learning. However, such learning may include instrumental (i.e., action response out-

Received March 13, 2003; revised July 29, 2003; accepted Aug. 6, 2003.

This work was supported by a programme grant from the Wellcome Trust (T.W.R) completed within the Medical Research Council Centre for Behavioral and Clinical Neuroscience. Y.C. was supported by Cambridge Cognition. We thank Dr. R. N. Cardinal for his help with programming and Dr. E. A. Murray for her comments on this manuscript.

Correspondence should be addressed to Dr. Y. Chudasama, Laboratory of Neuropsychology, National Institute of Mental Health, 49 Convent Drive, Building 49, Room 1B80, Bethesda, MD 20892-4415. E-mail: yogita@In.nimh.nih.gov.

Copyright $\odot 2003$ Society for Neuroscience $\quad 0270-6474 / 03 / 238771-10 \$ 15.00 / 0$ come) and pavlovian [i.e., conditional stimulus (CS)- unconditional stimulus] associations or depend on habit (stimulus-response) learning, all of which can potentially contribute to behavioral performance (Dickinson and Balleine, 1994). Operationally, discrimination learning is instrumental in that reinforcement is contingent on the subject choosing between the stimuli. However, because such discriminations also generally require a simple approach to one of the stimuli, classical conditioning (in the form of "autoshaping") (Brown and Jenkins, 1968) of approach and contact may sometimes be sufficient to generate the required response without the intervention of any instrumental process (Brown and Jenkins, 1968; Mackintosh, 1983).

Processes engaged during visual discrimination reversal learning are especially susceptible to OFC damage in primates (Jones and Mishkin, 1972; Rolls et al., 1994; Dias et al., 1996), whereas OFC-lesioned rats exhibit deficits in olfactory reversal learning (Brown and Bowman, 2002; Schoenbaum et al., 2002). The precise nature of these deficits is unclear because the subject not only has to learn the new stimulus-reward association but also inhibit prepotent responding to the originally reinforced stimulus. Iversen and Mishkin (1970) demonstrated that the monkey medial and lateral orbitofrontal cortices were, respectively, implicated in these two processes. 
Additional evidence for fundamental roles of different sectors of the PFC in associative learning and inhibitory control processes is emerging from rodent studies (Kolb et al., 1974; Eichenbaum et al., 1983; Granon and Poucet, 1995; Seamans et al., 1995; Brown and Bowman, 2002; Passetti et al., 2002). However, relatively little is known about the OFC as a ventral division of the rodent frontal cortex. Most studies have examined the effects of combined lesions of the ventral prelimbic and infralimbic cortices mainly on spatial tasks (Granon and Poucet, 1995; Seamans et al., 1995; Ragozzino et al., 1999). Furthermore, anatomical tracing studies have shown that the OFC and infralimbic cortex (ILC) have similar connections with autonomic structures (Neafsey, 1990; Hurley et al., 1991; Floyd et al., 2001), as well as sharing characteristic limbic and striatal connectivity (Öngür and Price, 2000).

Accordingly, we investigated the comparative roles of the rat OFC and ILC in the formation of pavlovian stimulus-reward associations (autoshaping) and reversal learning and also examined inhibitory control of previously reinforced discrimination habits. Thus, by preferentially damaging the OFC and the ILC using an excitotoxin that spares fibers of passage, this study elucidated the functional heterogeneity of the rodent ventral PFC.

\section{Methods and Materials \\ Subjects}

All subjects were male Lister Hooded rats (Charles River, Kent, UK), housed in pairs in a temperature-controlled room $\left(22^{\circ} \mathrm{C}\right)$ under diurnal conditions ( $12 \mathrm{hr}$ light/dark cycle). Rats were food deprived and maintained at $85 \%$ of their free-feeding weight throughout the experiment. All testing occurred at a regular time during the light period, and animals were 3 months of age ( $230-260 \mathrm{gm})$ at the start of behavioral training. All experimental procedures were subject to regulation by the United Kingdom Home Office (Project License PPL 80/1324).

\section{Surgical procedures}

Cytoarchitectonic divisions of the OFC and ILC were defined according to the stereotaxic atlas of Paxinos and Watson (1997). All animals were anesthetized by intraperitoneal injection of Avertin $(1 \mathrm{ml} / 100 \mathrm{gm})$ and placed in a stereotaxic head holder (David Kopf Instruments, Tujanga, CA) fitted with atraumatic ear bars. The scalp was retracted, and holes were drilled into the skull to expose the target region of the brain. The incisor bar was set at $-3.3 \mathrm{~mm}$. Lesions were made using a $1 \mu \mathrm{l}$ Hamilton syringe. OFC lesions were made using an additional glass micropipette (Harvard pipette puller) attached to the syringe to prevent mechanical damage to the most rostral and exposed orbitofrontal region. The neurotoxin was 0.09 m quinolinic acid (Sigma, Poole, UK) dissolved in a phosphate buffer, $\mathrm{pH}$ 7.0-7.2.

Animals of the OFC lesion group received three bilateral injections at the following coordinates: anteroposterior (AP), +4.0 $\mathrm{mm}$ from bregma; lateral (L), $\pm 0.8 \mathrm{~mm}$ from the midline; dorsoventral (DV), $-3.4 \mathrm{~mm}$ $(0.2 \mu \mathrm{l})$ below the dura; AP, $+3.7 \mathrm{~mm}$;, $\pm 2.0 \mathrm{~mm}$; DV,$-3.6 \mathrm{~mm}(0.3$ $\mu \mathrm{l})$; and AP, $+3.2 \mathrm{~mm}$;, $\pm 2.6 \mathrm{~mm}$; DV,$-4.4 \mathrm{~mm}(0.2 \mu \mathrm{l})$. For lesions of the ILC, animals received two bilateral injections of $0.4 \mu$ l of quinolinic acid at the following coordinates: $\mathrm{AP},+3.0 \mathrm{~mm}$ from bregma; $\mathrm{L}, \pm 0.7$ $\mathrm{mm}$ from the midline; $\mathrm{DV},-4.5 \mathrm{~mm}$ below the dura; and $\mathrm{AP},+2.5 \mathrm{~mm}$; $\mathrm{L}, \pm 0.7 \mathrm{~mm}$; DV,$-4.5 \mathrm{~mm}$. For both lesion groups, each injection was made over $3 \mathrm{~min}$, and the needle remained in place for an additional 3 min for dispersion before it was retracted. There were also surgical con-
B.

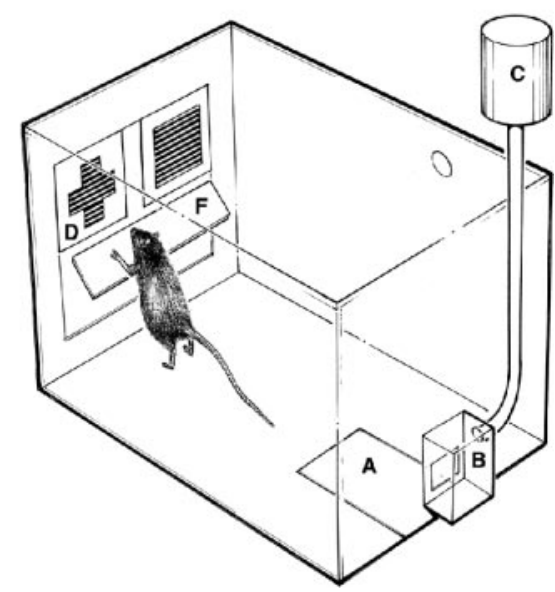
groups received the same surgical treatment as the OFC or ILC groups, except that they were infused with phosphate buffer.

\section{Apparatus}

Preliminary training and behavioral testing were performed in six automated touch-screen testing chambers (Cambridge Cognition, Cambridge, UK). The original design has been described previously (Bussey et al., 1994, 1997). The apparatus consisted of a testing chamber and a touch-screen unit housed within a sound-attenuating box. The box was fitted with a fan for ventilation and masking of extraneous noise. The inner chamber $(45 \times 30 \times 30 \mathrm{~cm}$; length $\times$ width $\times$ height $)$ consisted of three aluminum walls and one Perspex wall incorporating the door. The floor was also perforated and made of aluminum. The ceiling was constructed of clear Perspex. A $3 \mathrm{~W}$ house light was attached to a side wall. Located centrally at the rear of the chamber was a food magazine attached to a pellet dispenser. A panel light $(3 \mathrm{~W})$ illuminated the food magazine. Magazine entries were detected by photocells. A pressure-sensitive floor panel $(14.5 \times 10 \mathrm{~cm})$ was located directly in front of the food magazine, which was monitored by a microswitch. The stimuli were presented on a "touch-sensitive" open frame video display unit using TFT (Thin Film Transistor; Intasolve, Colchester, UK), which was located at the other end of the chamber. Both behavioral tasks were conducted in the same apparatus with modification as described below.

\section{Autoshaping}

The stimuli were white vertical rectangles displayed on the left and right of the video display unit screen measuring $6.5 \times 14 \mathrm{~cm}$. The chamber was equipped with a food magazine located centrally in front of the video display unit attached to a pellet dispenser. This pellet dispenser was situated outside the sound-attenuating box (Fig. 1). A left and right infrared beam was located just in front of the screen and at either side of the food magazine. A break in the beam was used to detect approaches to the left and right stimuli. The apparatus and online data collection were controlled by means of an Acorn computer system with software written in BASIC by Dr. R. N. Cardinal (Cambridge University, Cambridge, UK).

\section{Visual discrimination}

The touch screen was surrounded by an array of infrared beams that ran across the surface of the display. Contact with the screen was detected by the beams being broken, making the screen touch sensitive. A black, Perspex "mask" was attached to the face of the display unit $\sim 2 \mathrm{~cm}$ away from the surface of the display. The mask blocked access to the display unit except through response windows $(8 \times 9 \mathrm{~cm})$. Each response window was separated by a black Perspex divider to prevent accidental ap- 
proaches to the adjacent response window. A shelf extending $6.5 \mathrm{~cm}$ from the surface of the mask supported by springs was positioned just beneath the response windows $\sim 15 \mathrm{~cm}$ from the floor of the chamber. The stimuli consisted of a white rectangle and a white cross that were equated for luminance (Fig. $1 B$ ).

\section{Behavioral procedures \\ Autoshaping}

Pretraining. Rats were initially given two 30 min sessions in which they were allowed to habituate to the testing chamber and collect pellets from the central food magazine. The house light was illuminated, and pellets were delivered into the central magazine on a variable interval (VI) $40 \mathrm{sec}$ schedule. For the rat to familiarize itself with the floor panel, a food pellet was also delivered in the rear food magazine every time it stepped on the floor panel. After pretraining, the rear food magazine and pellet dispenser were never used. Animals were observed during this session to ensure that they were successfully retrieving and consuming pellets.

Acquisition. On the day after pretraining, animals were trained to associate stimuli with the delivery of a $45 \mathrm{mg}$ sucrose pellet (Bio-Serve, Frenchtown, NJ) dispensed in the central food magazine. A trial consisted of the presentation of $\mathrm{CS}^{+}$or $\mathrm{CS}^{-}$in a randomized order. One stimulus was designated the $\mathrm{CS}^{+}$, and the other was designated the $\mathrm{CS}^{-}$, which was counterbalanced across subjects. The session began with the illumination of the house light that was concomitant with the onset of a VI 10-40 sec schedule. This variable schedule was instituted because it ensured that the rats' approach behavior was not temporally mediated. Thus, after a VI 10-40 sec schedule, the rat was required to locate itself centrally on the floor panel at the rear of the chamber. This eliminated chance approaches to the stimuli and ensured equal stimulus sampling. The floor panel press that initiated the trial resulted in the presentation of either the $\mathrm{CS}^{+}$or the $\mathrm{CS}^{-}$stimulus for a duration of $10 \mathrm{sec}$. The $\mathrm{CS}^{+}$ signaled the delivery of a food pellet immediately after the offset of the stimulus. The $\mathrm{CS}^{-}$was never followed by food delivery. Acquisition consisted of 100 presentations of each of the $\mathrm{CS}^{+}$and $\mathrm{CS}^{-}$(two sessions of 50 presentations each on 2 consecutive days). When the rat broke either the left or right infrared beam that ran either side of the central food magazine, it was scored as an approach to that stimulus, and no additional approaches were scored during that stimulus presentation. The minimum time between presentations of the $\mathrm{CS}^{+}$and $\mathrm{CS}^{-}$was 10 sec. The maximum number of consecutive presentations of either the $\mathrm{CS}^{+}$or the $\mathrm{CS}^{-}$was two.

After acquisition, animals were placed on an omission test, during which the contingencies were altered such that approaches to the $\mathrm{CS}^{+}$ prevented the delivery of a food pellet. A tendency to approach to the $\mathrm{CS}^{+}$during this test indicates that learning during acquisition of autoshaping was attributable to pavlovian mechanisms rather than other forms of learning (Williams and Williams, 1969; Bussey et al., 1997). A "successful" omission trial was one in which the $\mathrm{CS}^{+}$was presented and the subject first approached the $\mathrm{CS}^{-}$or failed to approach either. There were 50 presentations of each of the $\mathrm{CS}^{+}$and $\mathrm{CS}^{-}$in one session.

The number of approaches to $\mathrm{CS}^{+}$and $\mathrm{CS}^{-}$were scored in blocks of 10 presentations, and difference scores $\left(\mathrm{CS}^{+}\right.$approaches $-\mathrm{CS}^{-}$approaches) were calculated. The mean latency to approach the $\mathrm{CS}^{+}$and $\mathrm{CS}^{-}$was calculated over 100 trials. Latencies to approach the stimulus were also examined, because this provided not only an indication of learning the association between the $\mathrm{CS}^{+}$and reward but also an index of locomotor capacity.

\section{Visual discrimination and reversal}

Pretraining. Rats were initially given one 30 min session in which they were allowed to habituate to the testing chamber. They were then given two 30 min sessions in which they were shaped to collect food pellets from the food magazine. During the first session, pellets were made freely available in the magazine panel with the magazine light on. During the second session, rats were trained to collect pellets that were delivered every time the rat pressed the floor panel. Once the animals were reliably retrieving a minimum of 50 pellets per session from the magazine, they were trained to respond to stimuli presented on the touch screen. During this procedure, a large white square was randomly presented in one of the two response windows. The square remained on the screen until the rat responded to it, after which the rat was rewarded with a pellet and the magazine light. Once the rat was able to obtain 50 reinforcements within $20 \mathrm{~min}$, the contingencies were introduced.

Acquisition. One stimulus was designated $\mathrm{S}^{+}$, and the other was designated $\mathrm{S}^{-}$; both stimuli were counterbalanced across subjects. The same pair of stimuli was presented on every trial, and the stimulus configuration for each trial (i.e., which stimulus was on the left and which stimulus was on the right) was determined pseudorandomly. The session began with the illumination of the house light. After a $5 \mathrm{sec}$ intertrial interval (ITI), the rat was required to locate itself on the pressure-sensitive floor panel. This resulted in the presentation of the stimulus. The rat was then required to approach the touch screen and make a response by selecting a stimulus and touching the screen with a nose poke. The stimulus remained on until the rat responded to it. A correct response to the $\mathrm{S}^{+}$was followed by the disappearance of the stimuli and the presentation of a 45 mg sucrose reward pellet concomitant with the illumination of the food magazine. The next trial commenced once the rat had obtained its reward, which was indicated by its breaking the photocell beam in the food magazine. This resulted in the food panel light being extinguished and the onset of the ITI. An incorrect response to the $\mathrm{S}^{-}$resulted in the disappearance of the stimuli and the house light being extinguished for a timeout period of $5 \mathrm{sec}$. Each session consisted of 100 trials. All animals were required to learn the correct, reinforced stimulus to a criterion of $85 \%$ on each of two consecutive sessions. As part of a correction procedure, after an incorrect choice, animals received the same stimulus configuration (i.e., the $\mathrm{S}^{+}$and $\mathrm{S}^{-}$remained in the same left-right locations) over successive trials until the rat had responded correctly.

Reversals. After acquisition of the discrimination, the reward contingency was reversed so that the previously nonrewarded stimulus was now the correct, reinforced stimulus (i.e., $\mathrm{S}^{+}$became $\mathrm{S}^{-}$and vice versa). Once the rat had attained a criterion of $85 \%$ on each of two consecutive sessions this reversal, the reward contingencies were reversed again. A total of two reversals were given. Each session consisted of 100 trials.

\section{Performance measures}

Several performance measures were calculated: (1) number of sessions to attain criterion performance; (2) average choice latency, which was the time from stimulus onset to the time the rat made a choice response; (3) average magazine latency, which was time from correct choice response to the time the rat entered the magazine to collect reward; and (4) percentage side bias, which was the number of responses either to the right or left response window depending on a particular animal's bias, expressed as a percentage of total trials for that session. These measures were calculated on the basis of noncorrection trials only.

The number of errors made during correction and noncorrection trials was also calculated. During correction trials, the same stimulus configuration was presented over consecutive trials until the animal made a correct response. Therefore, errors during these trials failed to dissociate repeated responses made to the visual stimulus from repeated responses on the side on which it was presented. These errors were classified as "correction errors."

The number of non-correction errors was further analyzed. After a "correct" response on a correction trial, the rat was presented with a noncorrection trial in which the stimulus configuration was spatially opposite to the previous correction trial. For example, if the animal responded correctly to correction trial $\mathrm{A}^{+} \mathrm{B}^{-}$, then the following noncorrection trial was $\mathrm{B}^{-} \mathrm{A}^{+}$. In addition, during consecutive noncorrection trials, the stimulus configuration was presented pseudorandomly. Therefore, during reversal learning, the number of errors made during noncorrection trials was analyzed according to two learning stages (Jones and Mishkin, 1972; Dias et al., 1996). All errors made within the reversal were classified as stimulus perseverative errors if the performance of the animals was significantly below chance-level performance (39\% correct for 100 trials). Errors that were committed between 40 and 85\% correct performance suggested that the animal had made a response away from the previously rewarded stimulus and continued to respond randomly until they shifted to the stimulus positively correlated with reward. These errors were classified as "learning errors." 
However, it was still possible that the animals' perseverative tendency during the reversal was influenced by factors that were not directly associated with the discriminability of the stimuli, i.e., errors made before the attainment of chance level performance may have been influenced by a spatial response bias. To separate stimulus perseveration from a response criterion, performance of noncorrection trials for the first three sessions were subjected to discrimination sensitivity index $\left(d^{\prime}\right)$ and response bias $(c)$ analysis derived from parametric signal detection models (Macmillan and Creelman, 1991).

Both $d^{\prime}$ and $c$ indices were calculated on the basis of the probability of hits $(h)$ and false alarms $(f)$ probabilities, which, in this twoalternative forced-choice task, were based on the number of times the rewarded stimulus was presented on the left (signal L) or right (signal $\mathrm{R}$ ) side and the number of times a response was made on the left (response L) or right (response R) side. Accordingly, hit = signal L/response L; miss $=$ signal $\mathrm{L} /$ response $\mathrm{R}$; false alarm $=$ signal $\mathrm{R} /$ response $\mathrm{L}$; and correct rejection $=$ signal $\mathrm{R} /$ response R. Thus, $p(h)=$ hits/hits + misses, whereas $p(f)=$ false alarms/false alarms + correct rejections.

According to the discrimination sensitivity in$\operatorname{dex},\left(d^{\prime}\right)=[z(h)-z(f)] / \sqrt{ } 2$, an animal that shows a positive $d^{\prime}$ score shows good discrimination and therefore makes "nonperseverative" errors. A negative $d$ ' score implies poor discrimination to the stimulus and suggests that the errors committed were stimulus perseverative errors. The criterion $(c)=-0.5[z(h)+z(f)]$ reflects the subjects' response bias. Thus, a value for $c$ of zero indicates a neutral criterion and represents no bias (Macmillan and Creelman, 1991).

\section{Data analysis}

Data for each variable were subjected to ANOVA using the SPSS statistical package, version 9.0 (SPSS, Chicago, IL). Data were initially explored using box plots and tests of homogeneity of variance so that outliers were identified, and skewed data, which violate the distribution requirement of the ANOVA, were transformed appropriately (arcsine, square root, or logarithmic) as recommended by Winer (1971). Homogeneity of variance across groups was assessed by the Mauchly sphericity test. When data sets significantly violated this requirement for a repeatedmeasures design, the Huynh-Feldt epsilon was used to calculate a more conservative $p$ value for each $F$ ratio. The criterion for statistical significance was a probability level of $p<0.05$. The between-subject factor for each experiment was lesion at two levels: sham controls (shams), ILC lesions, or OFC lesions. For the autoshaping task, within-subject factor included approaches made in blocks of 10 trials. For the visual discrimination task, the within-subject factor was reversal or stage of learning.

\section{Results}

Experiment 1a: effects of excitotoxic lesions of the OFC on acquisition of discriminated approach

Histological analysis

The cytoarchitectonic borders and nomenclature were taken from Paxinos and Watson (1997). Examination of the cresyl violet-stained sections revealed that two animals had extensive
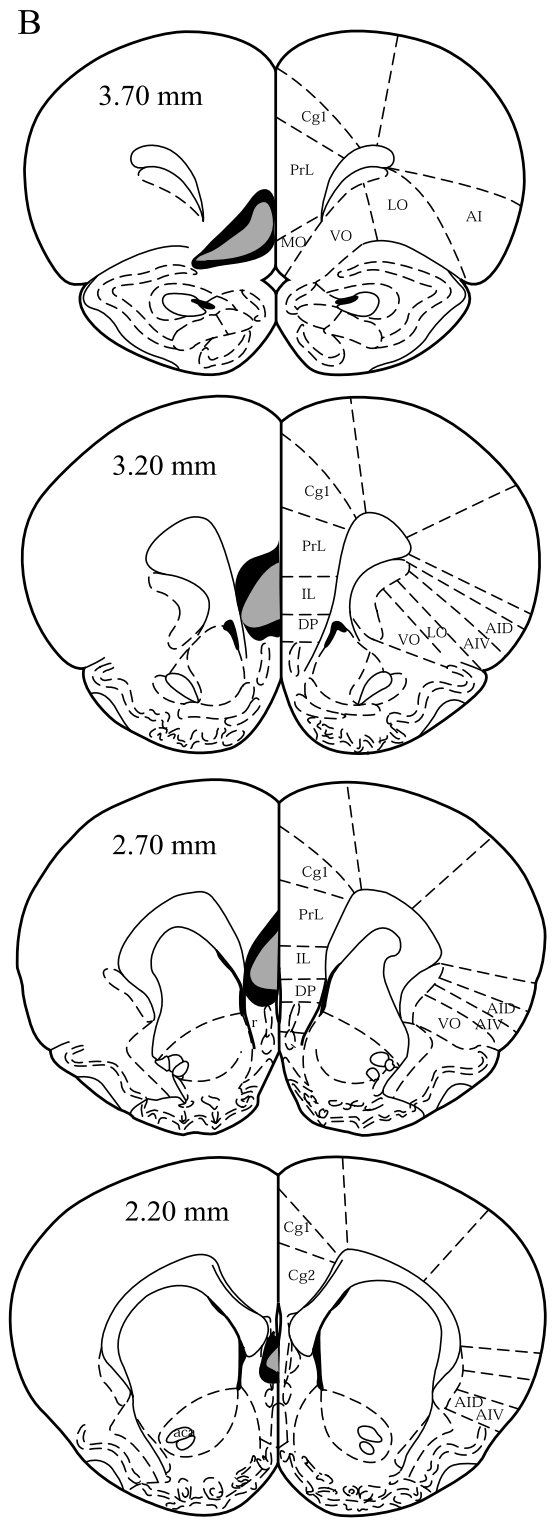

Diagrammatic reconstructions of coronal sections (Paxinos and Watson, 1997) showing the largest (black shading) and smallest (gray shading) extent of the OFC lesion from experiment $1 \mathrm{a}(A)$ and the ILC lesion from experiment $1 \mathrm{C}(B)$. Numbers in each section indicate the AP level anterior to bregma. AID, Dorsal agranular insular cortex; AIV, ventral agranular insular cortex; $\mathrm{Cg} 2$, cingulate cortex, area 2; DLO, dorsolateral orbital cortex.

damage that extended from the frontal pole and included the motor and somatosensory areas: the agranular insular cortex $(\mathrm{AI})$ and piriform region. These two animals were discarded from analysis. The remaining eight animals showed bilateral damage to the entire extent of the orbitofrontal region (Fig. 2A). The lesion started at bregma +4.7 and included the most medial $(\mathrm{MO})$ and ventral orbital (VO) regions. At this most rostral extent, the lesion encroached into the prelimbic cortex (PrL). The lesion then continued to include the ventral and lateral orbital (LO) cortex at bregma +3.2 , where the most lateral limits of the ILC was also damaged (Fig. 2B), although for the most part, the ILC was mostly spared, as was the dorsal peduncular (DP) and the PrL cortex. At its most caudal extent (bregma +2.7 ), the lesion included the VO and LO and the most ventral AI cortex. Despite the close proximity of the striatum to the OFC at its most caudal level, no gliosis or calcification was observed in the striatum. One sham-operated rat died as a result of a respiratory infection dur- 
A

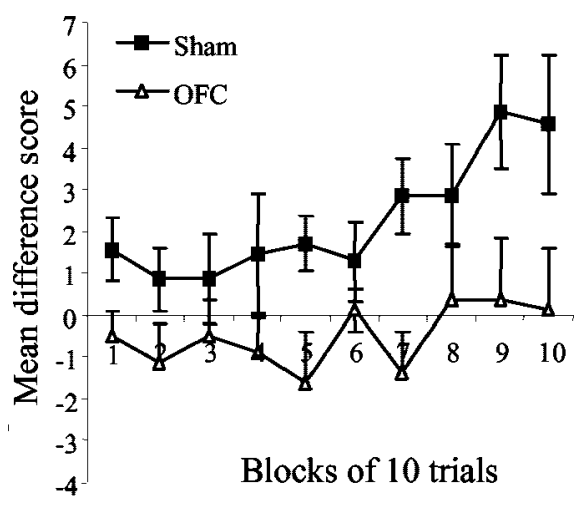

B

Omission

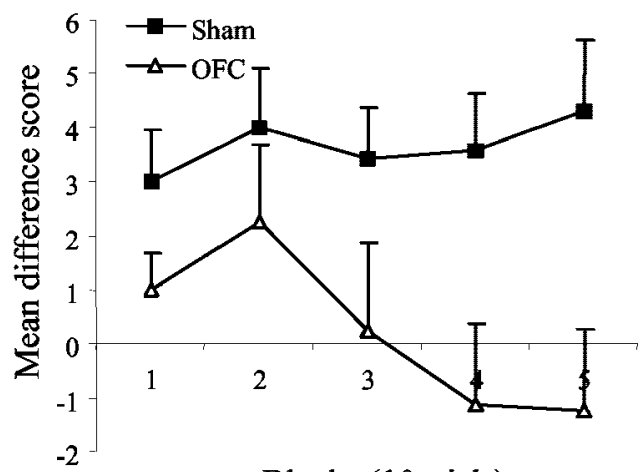

Blocks (10 trials)

Figure 3. Mean \pm SEM difference scores of sham controls and animals with OFC lesions on discriminated approach: $A$, acquisition; $B$, omission test.

ing postoperative recovery. Thus, the final group numbers for this study were as follows: shams, 7; and OFC, 8.

\section{Behavioral results}

Approaches to $\mathrm{CS}^{+}$and approaches to $\mathrm{CS}^{-}$were analyzed for the sham- and OFC-lesioned groups across 10 blocks of 10 stimulus presentations. There was a tendency for the OFC group to make fewer approaches $\left(F_{(1,13)}=3.56 ; p=0.08\right.$; means, sham 37, OFC $20)$, but they were no different from the sham controls in terms of approaches to the $\mathrm{CS}^{-}\left(F_{(1,13)}=2.26 ; p>0.05\right)$. A comparison of the difference scores (Fig. $3 A$ ) revealed that, although all animals showed improved discriminated approach behavior over blocks of trials $\left(F_{(5,68)}=2.32 ; p<0.05\right)$, the OFC group was significantly impaired relative to the sham group $\left(F_{(1,13)}=11.56 ; p<0.01\right)$. The OFC group was also slower to approach the stimuli $\left(F_{(1,13)}=\right.$ 4.70; $p<0.05$; means in centiseconds for $\mathrm{CS}^{+}$, shams 520 , OFC 608 ; $\mathrm{CS}^{-}$, shams 530, OFC 606) and were also slower to collect food reward $\left(F_{(1,13)}=4.92 ; p<0.05\right.$; means in centiseconds, shams 395, OFC 470).

During the omission test, the OFC group made significantly fewer approaches to the $\mathrm{CS}^{+}\left(F_{(1,13)}=4.61 ; p<0.05\right)$. An analysis of the difference scores confirmed that the OFC group was significantly impaired in discriminated approach $\left(F_{(1,13)}=6.11\right.$; $p<0.05$ ) (Fig. $3 B$ ). Animals with OFC lesions were slower to approach the $\mathrm{CS}^{+}\left(F_{(1,13)}=9.72 ; p<0.01\right)$ but were not slower to collect reward food pellets $\left(F_{(1,13)}=0.21 ; p>0.05\right)$.

Therefore, lesions of the OFC disrupted the acquisition of autoshaping as characterized by a reduction in the number of approaches to the $\mathrm{CS}^{+}$and retarded approach latencies. This implies that the OFC contributes to the direct learning of the stimulus-reward association. To explore the possible involvement of the OFC in the expression of the learned association, the effects of OFC lesions were examined after initial training on the task.

\section{Experiment 1b: effects of excitotoxic lesions of the OFC on retention and performance of discriminated approach}

Histological analysis

Examination of the cresyl violet-stained sections revealed that, of the nine animals initially assigned to the OFC group, three animals presented with either an incomplete lesion or too extensive a lesion. These animals were discarded from analysis. The remaining lesions were found to be centered on the appropriate target region of the brain and were similar to those obtained in experiment 1a. All seven sham control animals showed no neu- ronal damage. The final group numbers were as follows: sham, 7; OFC, 6.

\section{Preoperative performance}

All animals learned the discriminated approach to a high level before surgery. Final difference scores for the future sham and lesion groups were $\left(F_{(1,11)}=0.02 ; p>0.05\right.$; mean score, shams 15.2, OFC 16.6). In addition, the number of approaches made by the animals designated to the sham control group in this study did not differ from the number of approaches made by the sham control animals postoperatively in experiment $1 \mathrm{a}\left(F_{(1,12)}=0.49\right.$; $p>0.05)$.

\section{Postoperative performance (blocks 1-10)}

There was a transient deficit during the first block in the OFC group $\left(F_{(1,12)}=6.39 ; p<0.05\right)$. However, discriminated performance was not significantly different between the groups from that point $\left(F_{(1,11)}=1.17 ; p>0.05\right)$, reaching asymptote difference score values by block 10 (means, sham 7.5, OFC 6.0). On the omission test, there was a tendency for the OFC group to show significantly fewer approaches $\left(F_{(1,11)}=4.80 ; p=0.051\right.$; mean difference scores collapsed across block, sham 33, OFC 24). There were no differences between groups in latencies to approach the stimulus or collecting food reward ( $p>0.05$ ).

In summary, OFC lesions did not impair performance of the preoperatively trained autoshaped response, but there was a detrimental effect on the omission test.

\section{Experiment 1c: effects of excitotoxic lesions of the ILC on discriminated approach}

Histological analysis

Figure $2 B$ presents a diagrammatic representation of the extent of the lesions in the ILC-lesioned group. After histological analysis, one animal from the ILC group was found to have a unilateral lesion. Another two animals were found to have very large lesions, which included most of the rostral prelimbic cortex but left the ILC intact. The data from these three animals were therefore excluded from subsequent behavioral analysis. The remaining seven animals showed evidence of bilateral ILC damage, with almost complete neuronal loss. The lesion extended from approximately AP $+3.7 \mathrm{~mm}$ to $\mathrm{AP}+2.2 \mathrm{~mm}$ from bregma. All animals showed slight sparing of the ILC at the most lateral and caudal limits of this region. The lesion was found to encroach ventrally into the PrL cortex and dorsally into the dorsal pedun- 
A

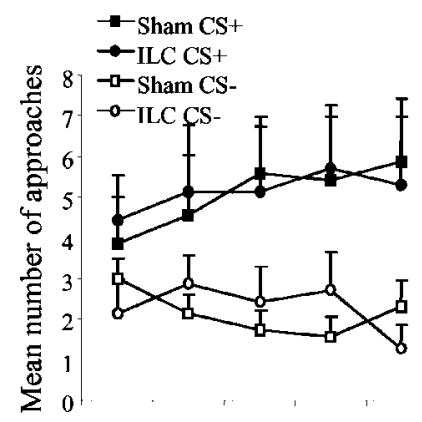

$\stackrel{6}{6} \stackrel{7}{8} \stackrel{9}{9} \quad 10$
$\mathbf{B}$

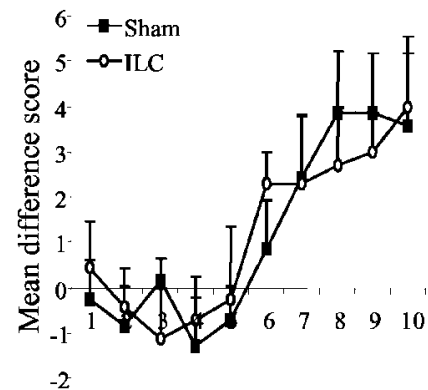

Blocks (10 trials)

Figure 4. Mean \pm SEM performance of ILC on acquisition of discriminated approach (autoshaping): $A$, number of approaches to the $C S^{+}$and $C S^{-}$for last five blocks of trials; $B$, difference scores.

cular cortex. In the sham group, one animal showed neuronal damage in the dorsal anterior cingulate $(\mathrm{Cg} 1)$ and PrL region of the cortex. This animal was excluded. The final numbers in each group for behavioral analyses were as follows: shams, 7; ILC, 7.

\section{Behavioral results}

The mean numbers of approaches to the $\mathrm{CS}^{+}$and $\mathrm{CS}^{-}$for the last five blocks are presented in Figure $4 A$. A comparison of the difference scores (Fig. $4 B$ ) revealed that both groups of animals showed equivalently improved discriminated approach behavior over blocks of trials $\left(F_{(1,12)}=0.008 ; p>0.05\right)$. However, during the omission test, although the sham controls and the ILC group did not differ in the number of approaches to the $\mathrm{CS}^{+}\left(F_{(1,12)}=\right.$ $2.41 ; p>0.05$; mean approaches, sham 33, ILC 22), an analysis of the difference scores showed a tendency for these animals to be significantly different from shams $\left(F_{(1,12)}=4.60 ; p=0.053\right.$; mean difference score for block 5, sham 4.1, ILC 2.1), but there was no lesion $\times$ block interaction $(p>0.05)$. Approach and magazine latencies were not affected ( $p>0.05)$. Therefore, ILClesioned animals exhibited good discriminated approach during acquisition but were sensitive to the effects of reward omission.

Experiment 2a: effects of excitotoxic lesions of the OFC cortex on visual discrimination and reversal learning

Histological analysis

In this different experimental group of OFC-lesioned animals, examination of the cresyl violet-stained sections revealed that two of these animals presented with an incomplete, unilateral lesion. A third animal showed extensive damage that included most part of the medial frontal and motor cortex. These animals were discarded from analysis. In all other cases, the lesions were similar to those obtained in experiment 1a. The OFC lesion started at the rostral pole and included the medial orbital cortex and extended to the most caudal level, where it included the VO and LO cortex. The final numbers for analysis were as follows: sham, 7; OFC, 10.

\section{Behavioral results}

Acquisition. There was no differential effect of lesion in terms of the number of sessions to acquire the visual discrimination $\left(F_{(1,15)}=0.42 ; p>0.05\right.$; means, sham 11, OFC 12), the number of errors committed to reach criterion performance $\left(F_{(1,15)}=0.14\right.$; $p>0.05$; means, sham 387, OFC 378), or percentage side bias $\left(F_{(1,15)}=0.19 ; p>0.05\right.$; means, sham $48.6 \%$, OFC 50.1\%). The OFC group was slower to make a response $\left(F_{(1,15)}=6.42 ; p<\right.$
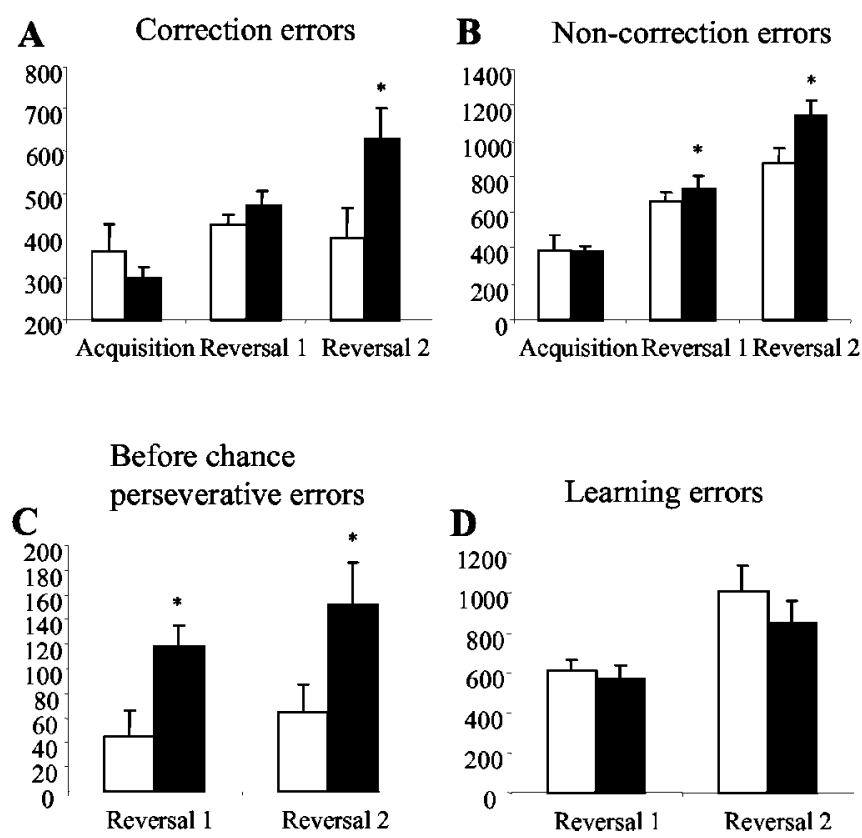

Figure 5. Performance of sham controls (white bars) and OFC lesion (black bars) group on visual discrimination and reversal learning. Each error bar represents mean $\pm \mathrm{SEM}: A$, errors committed during correction trials; $B$, errors committed during noncorrection trials; $C$, stimulus perseverative errors committed before chance performance; $D$, learning errors committed after chance performance.

0.05; means in centiseconds, sham 534, OFC 704), although magazine latencies remained unaffected $\left(F_{(1,15)}=0.11 ; p>0.05\right.$; means in centiseconds, sham 133, OFC 114).

Reversals. Although overall more sessions were required to reach criterion during the second reversal $\left(F_{(1,15)}=8.29 ; p<\right.$ $0.01)$, the lesion groups did not differ on this measure $\left(F_{(1,15)}=\right.$ $0.23 ; p>0.05)$, and there was no lesion $\times$ reversal interaction $(p>0.05)$. Figure 5, $A$ and $B$, shows the number of correction and noncorrection errors, respectively, committed by animals during acquisition and the two reversals of the discrimination. Analysis of the number of correction errors committed over two reversals revealed a lesion $\times$ reversal interaction $\left(F_{(1,15)}=6.82\right.$; $p<0.05$ ) that was attributable to the OFC group making significantly more errors relative to sham controls during reversal 2 $\left(F_{(1,15)}=4.36 ; p<0.05\right)$. There was no difference in the number of correction errors committed during reversal $1(p>0.05$; means, reversal 1, sham 425, OFC 469; reversal 2, sham 391, OFC $629)$. In addition, although all animals made more noncorrection trial errors during reversal learning $\left(F_{(1,15)}=25.10 ; p<0.001\right)$, the OFC group committed significantly more errors relative to sham controls $\left(F_{(1,15)}=4.39 ; p<0.05\right)$ (Fig. $\left.5 B\right)$. No lesion $\times$ reversal interaction was observed.

The noncorrection errors were further analyzed according to the type of errors made during two stages of learning (Fig. $5 C, D$ ): before chance perseverative errors $(<39 \%$ correct $)$ and at and above chance learning errors ( $\geq 40-85 \%$ correct). Overall, more errors were committed during reversal 2 during both stages of learning $\left(F_{(1,15)}=172.57 ; p<0.001\right)$. In addition, both groups of animals made more learning errors $\left(F_{(1,15)}=20.54 ; p<0.001\right)$ relative to perseverative errors. The pattern of errors committed during the two stages of learning shows that the OFC group made more perseverative errors before chance performance was attained $\left(F_{(1,15)}=9.53 ; p<0.01\right)$. In contrast, the OFC group was not different from shams in committing learning errors $\left(F_{(1,15)}=\right.$ 

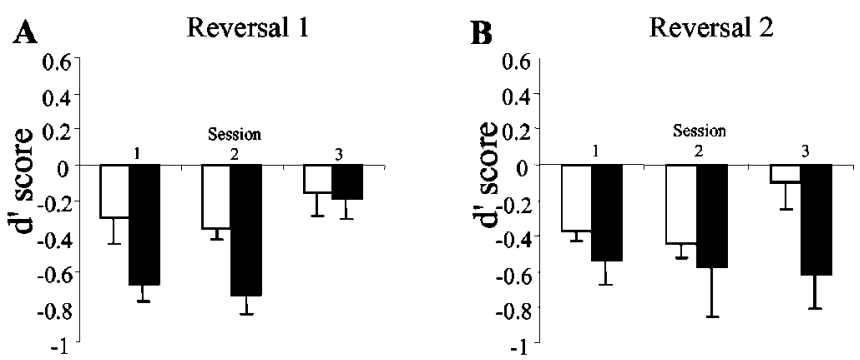

C Reversal 1

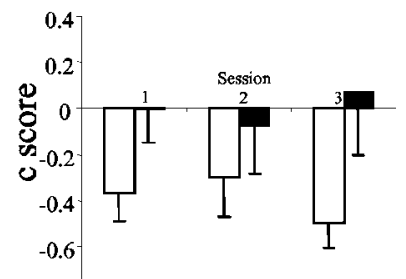

$-0.8$

Figure 6. Mean \pm SEM discrimination sensitivity ( $d$ ' score) and responsivity (c score) index for sham controls (white bars) and OFC lesion (black bars) groups for each reversal during the first three sessions: $A, d^{\prime}$ score for reversal $1 ; B, d^{\prime}$ score for reversal $2 ; C$, c score for reversal $1 ; D$, cscore for reversal 2.

$1.00 ; p>0.05)$. If anything, OFC-lesioned animals made fewer errors during each reversal at the second stage of learning.

Discrimination sensitivity index ( $\left.\mathrm{d}^{\prime}\right)$ and responsivity criterion (c) To examine further the extent of stimulus perseveration of the OFC-lesioned animals, the noncorrection errors were analyzed according to $d^{\prime}$ scores of discrimination and $c$ scores of response bias. Figure $6, A$ and $B$, shows the discrimination sensitivity scores for the first three sessions for each reversal. During the first reversal, the OFC group showed significantly lower $d^{\prime}$ scores (more negative) compared with the sham group $\left(F_{(1,15)}=7.50\right.$; $p<0.01)$ across sessions $\left(F_{(2,30)}=5.47 ; p<0.001\right)$. For reversal 2 , however, whereas the sham animals exhibited improved discrimination by session 3 ( $d^{\prime}$ score of -0.10$)$, the OFC group was still impaired ( $d^{\prime}$ score of -0.57 ) (Fig. $6 B$ ), but there was no significant effect of lesion $\left(F_{(1,15)}=1.89 ; p>0.05\right)$.

Figure $6, C$ and $D$, shows the mean $c$ scores that represent an index of response bias. Although the OFC group showed lower $c$ scores (less negative) than the sham controls, there was no differential effect of lesion on this measure $\left(F_{(1,15)}=3.09 ; p=0.09\right)$. During the second reversal (Fig. $7 D$ ), the two groups of animals showed equal levels of bias $\left(F_{(11,15)}=0.018 ; p>0.05\right)$.

Latency measures. Both groups of animals improved response latencies with time $\left(F_{(1,15)}=12.71 ; p<0.01\right)$, but, unlike during discrimination acquisition, there was no significant effect of lesion on this measure $\left(F_{(1,15)}=2.55 ; p>0.05\right.$; means in centiseconds for reversal 1, sham 393, OFC 309; reversal 2, sham 454, OFC 373). Magazine latency was unaffected $(p>0.05)$.

Percentage bias. There was no significant effect of lesion on this measure ( $p>0.05$; means, reversal 1, sham $47.7 \%$, ILC $48.6 \%$; reversal 2, sham $47.6 \%$, ILC $48.7 \%$ ), nor was there an effect or reversal or a lesion $\times$ reversal interaction $(p>0.05)$.

To summarize, although rats with OFC lesions were not impaired in acquiring the visual discrimination, these animals were highly perseverative during both reversals. This perseverative deficit was in the absence of any motivational, motoric, or response bias deficit.

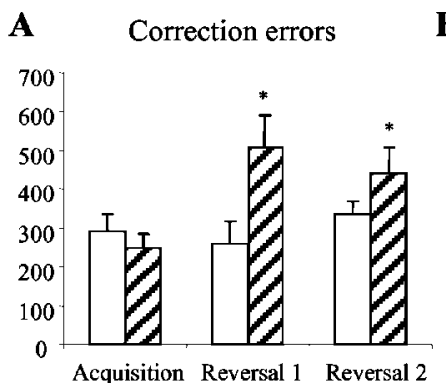

B Non-correction errors
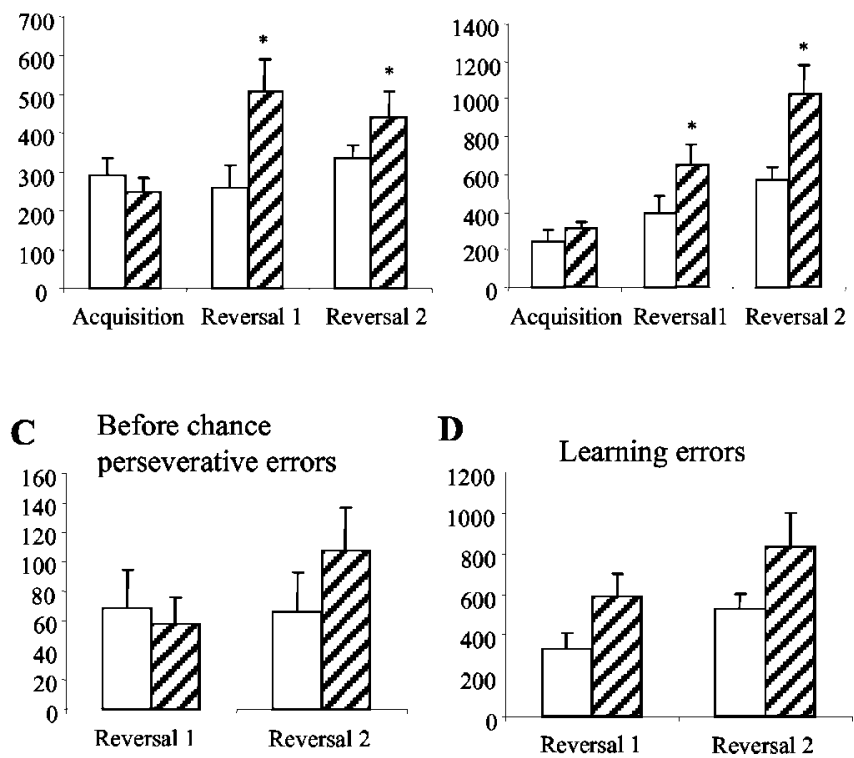

Figure 7. Mean \pm SEM performance of sham controls (white bars) and ILC lesion (hatched bars) group on visual discrimination and reversal learning: $A$, errors committed during correction trials; $B$, errors committed during non-correction trials; $C$, stimulus perseverative errors committed before chance performance; $D$, learning errors committed after chance performance.

Experiment 2b: effects of excitotoxic lesions of the ILC on visual discrimination and reversal learning

Histological analysis

Examination of the cresyl violet-stained sections revealed that two of the sham-lesioned animals showed evidence of frontal cortical damage, particularly around the tract mark. A third sham-operated rat died as a result of a respiratory infection. Thus, three sham operated rats were excluded from behavioral analysis. In the ILC group, one animal was found to have a unilateral lesion, and another was found to have a small, incomplete lesion. These two animals from the ILC group were also excluded from behavioral analysis. In all other cases, the area of the lesion was centered on the appropriate target region and was similar to those obtained in experiment 1c. The final numbers for each group in this experiment were as follows: shams, 6; ILC lesion, 8.

\section{Behavioral results}

Acquisition. ANOVA revealed that there was no significant effect of the ILC lesion on the acquisition of the visual discrimination task in terms of the number of sessions $\left(F_{(1,13)}=3.29 ; p>0.05\right.$; means, shams 8 , ILC 11) or the number of errors $\left(F_{(1,13)}=1.76\right.$; $p>0.05$; means, shams 244 , ILC 318$)$ to reach criterion. However, ILC lesions produced fast response latencies compared with sham controls $\left(F_{(1,13)}=5.80 ; p<0.05\right.$; means in centiseconds, sham 476; ILC 347). These animals were also significantly faster at collecting food reward $\left(F_{(1,13)}=9.01 ; p<0.01\right.$; means, shams 290 , ILC 159). No other effects were obtained ( $p>0.05)$.

Reversals. Overall, animals required more sessions to reach criterion performance during the second reversal $\left(F_{(1,12)}=19.6\right.$; $p<0.01)$, but the ILC group required more sessions relative to sham controls $\left(F_{(1,12)}=4.45 ; p<0.05\right.$; means, reversal 1 , sham 12, ILC 19; reversal 2, sham 16, ILC 29). These animals also committed more correction errors $\left(F_{(1,12)}=7.98 ; p<0.05\right)$ (Fig. $7 A)$ and noncorrection errors $\left(F_{(1,12)}=6.01 ; p<0.05\right)$ (Fig. $\left.7 B\right)$ relative to the sham group. 
The number of noncorrection error type during the two stages of learning (before chance perseveration and learning errors) for each reversal is shown in Figure 7, $C$ and $D$. Overall, more errors were committed during reversal 2 than reversal 1 for both stages of learning $\left(F_{(1,12)}=36.49 ; p<0.001\right)$. Animals also made more learning errors $\left(F_{(1,12)}=23.12 ; p<0.001\right)$ compared with perseverative errors. There was no stage $\times$ group interaction $(p>$ 0.05). However, as Figure 7D shows, the ILC group committed more learning errors relative to sham controls (means for reversal 1, sham 330, ILC 558; reversal 2, sham 527, ILC 832), although this difference just failed to reach significance $\left(F_{(1,12)}=3.95 ; p=\right.$ $0.07)$.

Latency measures. ILC-lesioned animals showed faster response latencies (means in centiseconds, reversal 1, sham 442, ILC 334; reversal 2, sham 381, ILC 303), although these differences were not statistically supported $\left(F_{(1,12)}=1.98 ; p>0.05\right)$. In addition, animals with ILC lesions tended to be faster at collecting food reward $\left(F_{(1,12)}=4.49 ; p=0.056\right.$; means in centiseconds, reversal 1, sham 179, ILC 106; reversal 2, sham 181, ILC 123).

Percentage bias. There was no significant effect of lesion on this measure ( $p>0.05$; means, reversal 1 , sham $48 \%$, ILC $47 \%$; reversal 2 , sham $46 \%$, ILC $48 \%$ ).

To summarize, rats with ILC lesions were able to acquire the visual discrimination task as normally as sham controls but tended to commit more learning errors during the reversals. This deficit was accompanied by very fast latencies, particularly when collecting food reward.

\section{Discussion}

The present study has shown dissociable effects of selective damage to the rat OFC and ILC on different aspects of discrimination reversal learning and pavlovian appetitive conditioning. The OFC group was profoundly impaired in learning to approach cues predictive of food (autoshaping) but remained unimpaired in instrumental simultaneous visual discrimination learning. The OFC lesion, however, did impair aspects of instrumental learning, as characterized by excessive perseverative responding during the early stages of reversal learning. In contrast, the ILC-lesioned rats did not perseverate but showed a (nonsignificant) tendency instead to be impaired in learning the new discrimination.

The deficit in appetitive pavlovian conditioning in the OFC group was accompanied by slowed approach latencies and retarded magazine responses. However, it is difficult to argue that the OFC rats had fundamental performance deficits because they consumed all of the pellets in the autoshaping task and acquired the instrumental visual discrimination task normally. Furthermore, only transient deficits were observed after OFC lesions on preoperatively acquired discriminative approach behavior. Thus, the most parsimonious explanation is of impaired pavlovian conditioning. Although evidence from the omission test showed that performance in the sham-operated rats was mostly under control of pavlovian contingencies, the OFC group was sensitive to the omission of food reinforcers after approach responses, suggesting that their behavior was under a degree of instrumental control (Brown and Jenkins, 1968). Together with the lack of deficit in the instrumental visual discrimination task, these data implicate the OFC in pavlovian rather than instrumental learning processes.

These results also add an important node to a neural network encompassing the anterior cingulate cortex, central nucleus of the amygdala, and core subregion of the nucleus accumbens (Parkinson et al., 2000a,b; Cardinal et al., 2002), which has been implicated in the learning of pavlovian approach behavior. Of these regions, only the central nucleus of the amygdala has shown any specificity for the acquisition stage of this form of appetitive conditioning, as distinct from its expression (Cardinal et al., 2002). Furthermore, apart from its lateral orbital portion, the OFC projects to the entire ventromedial region of the striatum (Phillipson and Griffiths, 1985; Berendse et al., 1992; Brog et al., 1993); it is only the ILC that has a unique projection to the accumbens shell (Hurley et al., 1991; Öngür and Price, 2000). This evidence of plasticity within the OFC is consistent with hypotheses about a special role for the OFC in stimulus-reward learning (Rolls, 1996, 2000; Schoenbaum et al., 2000).

In contrast, there was no evidence of any major deficit in learning pavlovian discriminative approach in the ILC group, although their performance in the omission test suggested that their approach behavior was under somewhat different control than in the sham-operated controls. A more specific impairment emerged in the ILC group during the reversal stage when the ILC group took significantly more sessions to reach criterion. Furthermore, they tended to make more errors to reach the learning criterion after, rather than before, chance performance had been attained, thus tending (though nonsignificantly) toward an impairment in new stimulus-reward learning rather than in the inhibitory control of previously reinforced responses (Jones and Mishkin, 1972). However, this fails to explain why these animals were not impaired in the initial acquisition of instrumental visual discrimination. One possibility is that these animals were able to encode the relevant features or perceptual dimensions of the discriminanda during initial conditioning but then failed to use that attentional learning to reverse rapidly thereafter (Sutherland and Mackintosh, 1971; Bussey et al., 1997). This may explain the lack of effect during acquisition of the instrumental discrimination. Nonetheless, this result is similar to findings of effects of medial PFC lesions, using the same reversal learning paradigm (Bussey et al., 1997), and it now seems likely that this medial PFC substrate includes the ILC.

Although OFC-lesioned rats were not impaired in visual discrimination learning or in the late phases (i.e., "learning" phases) of reversal learning, there was nevertheless a very large deficit in the early stages of reversal. This was characterized by an inability to inhibit previously reinforced responses, leading to perseveration, a finding reminiscent of previous studies in monkeys (Iversen and Mishkin, 1970; Jones and Mishkin, 1972; Dias et al., 1996) and more recently in olfactory reversal learning in rats (Schoenbaum et al., 2002). Furthermore, the present findings implicate a dissociation of the perseverative deficit from one of new learning in the context of instrumental discrimination. Importantly, the OFC animals did not have difficulty in learning the new visual stimulus-reward association once the perseverative tendency was overcome. Indeed, these animals made fewer errors than controls during the late phase of learning. This implies a selective "executive" impairment of response control. Overall, it might be concluded that the selective perseverative impairment of the OFC-lesioned rats was in failing to inhibit an instrumental response, possibly exacerbated by the effects of proactive interference from the previously established association, leading to an enhanced expression of a stimulus-response (or "visual") habit that is impervious to changes in the value of reinforcement (Mishkin 1964; Dickinson and Balleine, 1994; Balleine and Dickinson, 1998). This is consistent with data showing that monkeys with frontal (ventral and orbital) lesions are impaired in the ac- 
quisition of new visuomotor associations, particularly when having to choose among three equally reinforced responses (Bussey et al., 2001). Importantly, these data also suggest that mnemonic aspects of instrumental learning tasks may be a critical component in measures of behavioral flexibility.

However, the OFC-lesioned rats were not globally "disinhibited" (cf. Fuster, 1989). First, although they made more responses to the $\mathrm{CS}^{-}$in the autoshaping paradigm, they also made fewer approaches to the $\mathrm{CS}^{+}$. Second, they exhibited faster extinction on the omission test. Third, response latencies were slower in the acquisition of the discriminated approach and the visual discrimination tasks, although both response and magazine latencies were normal at other stages of the visual reversal paradigm. The OFC-lesioned rats also exhibit increased perseveration in other settings, notably the five choice serial reaction time task (5CSRTT), but again in the absence of generalized disinhibition, because "impulsive," premature responding is relatively normal in this test (Chudasama et al., 2003).

Compared with the OFC group, the ILC-lesioned group exhibited an opposite tendency in disrupted response control, toward faster responding, in the absence of perseveration. This was evident in terms of both choice and magazine latencies in the acquisition of the visual discrimination task. This rapid responding might be related to the distinct form of disinhibition shown by the ILC rats in the 5-CSRTT, enhanced premature, but not perseverative, responding (Chudasama et al., 2003). Overall, the invigoration of performance after ILC lesions in the rat is reminiscent of motivational changes described in dogs after medial orbital lesions as "drive disinhibition" (Brutkowski and Memple, 1961; Brutkowski and Dabrowska, 1963; Brutkowski, 1964).

The dissociation of behavioral effects observed after lesions of the OFC and ILC on autoshaping and discrimination reversal learning provides new insights into the functional organization of the rodent frontal cortex. In particular, our data suggest that distinct regions of the rodent ventral frontal cortex mediate independent but complementary processes encompassing different forms of associative learning and response control. These data parallel, to some extent, similar distinctions made in other species for lateral and medial sectors of the OFC (Brutkowski, 1964; Iversen and Mishkin, 1970; Jones and Mishkin, 1972) and support the more general contention that executive functions are readily localizable in the frontal cortex and lie within discrete frontal regions (Passetti et al., 2002; Aron et al., 2003). To what extent the deficits in associative learning and response inhibition, both within and between, these regions are inter-related is unclear, but there is clearly some reciprocity in the overall control of behavioral output. For example, the OFC normally presumably promotes pavlovian learning but suppresses instrumental "habits," whereas the ILC promotes instrumental discrimination learning and suppresses impulsive responding that may arise from an overexpression of pavlovian influences, such as approaching the food magazine.

In terms of clinical significance, disturbances in associative learning and response inhibition after damage to the OFC are integral to virtually all disorders of "acquired sociopathy" (Bechara et al., 1998, 1999; Rogers et al., 1999) after frontal lobe injury, attention deficit-hyperactive disorders (Casey et al., 1997), and frontal lobe dementia (Rahman et al., 1999), as well as other impulse control syndromes, including obsessive compulsive disorder (Baxter, 1990) and drug addiction (Volkow and Fowler, 2000).

\section{References}

Aron AR, Fletcher PC, Bullmore ET, Sahakian BJ, Robbins TW (2003) Stopsignal inhibition disrupted by damage to right inferior frontal gyrus in humans. Nat Neurosci 6:115-116.

Balleine B, Dickinson A (1998) Goal-directed instrumental action: contingency and incentive learning and their cortical substrates. Neuropharmacology 37:407-419.

Baxter LR (1990) Brain imaging as a tool in establishing a theory of brain pathology in obsessive compulsive disorder. J Clin Psychol 52:22-25.

Bechara A, Damasio AR, Damasio H, Anderson SW (1994) Insensitivity to future consequences following damage to human prefrontal cortex. Cognition 50:7-15.

Bechara A, Domasio H, Tranel D, Anderson SW (1998) Dissociation of working memory from decision-making within human prefrontal cortex. J Neurosci 18:428-437.

Bechara A, Domasio H, Domasio AR, Lee GP (1999) Different contributions of the human amygdala and ventromedial prefrontal cortex to decision-making. J Neurosci 19:5473-5481.

Berendse HW, Galis-de GraffY, Groenewegen HJ (1992) Topographical organisation and relationship with ventral striatal compartments of prefrontal corticostriatal projections in the rat. J Comp Neurol 316:314-347.

Brog JS, Salyapongse A, Deutch AY, Zahm DS (1993) The patterns of afferent innervations of the core and shell in the "accumbens" part of the rat ventral striatum: immunohistochemical detection of retrogradely transported fluoro-gold. J Comp Neurol 338:255-278.

Brown PL, Jenkins HJ (1968) Autoshaping of the pigeon's keypeck. J Exp Anal Behav 11:1-8.

Brown VJ, Bowman EM (2002) Rodent models of prefrontal function. Trends Neurosci 25:340-343.

Brutkowski S (1964) Prefrontal cortex and drive inhibition. In: The frontal granular cortex and behaviour (Warren JM, Akert K, eds), pp 242-270. New York: McGraw-Hill.

Brutkowski S, Dabrowska J (1963) Disinhibition after prefrontal lesions as a function of duration of intertribal intervals. Science 139:505-506.

Brutkowski S, Memple E (1961) Disinhibition of inhibitory conditioned responses following selective brain lesions in dogs. Science 134:2040-2041.

Bussey TJ, Muir JL, Robbins TW (1994) A novel automated touchscreen procedure for assessing learning in the rat using computer graphic stimuli. Neurosci Res Commun 15:103-110.

Bussey TJ, Everitt BJ, Robbins TW (1997) Dissociable effects of cingulate and medial frontal cortex lesions on stimulus-reward learning using a novel pavlovian autoshaping procedure for the rat: implications for the neurobiology of emotion. Behav Neurosci 111:1-12.

Bussey TJ, Wise SP, Murray EA (2001) The role of ventral and orbital prefrontal cortex in conditional visuomotor learning and strategy use in rhesus monkeys (Macaca mulatta). Behav Neurosci 115:971-982.

Butter CM (1969) Impairments in selective attention to visual stimuli in monkeys with inferotemporal and lateral striate lesions. Brain Res 12:374-383.

Cardinal RN, Parkinson JA, Lachenal G, Halkerston KM, Rudarakanchana N, Hall J, Morrison CH, Howes SR, Robbins TW, Everitt BJ (2002) Effects of selective excitotoxic lesions of the nucleus accumbens core, anterior cingulate cortex, and central nucleus of the amygdala on autoshaping performance in rats. Behav Neurosci 116:553-567.

Casey BJ, Trainor RJ, Orendi JL, Schubert AB, Nystrom LE, Giedd JN, Castellanos X, Haxby JV, Noll DC, Cohen JD, Forman SD, Dahl RE, Rapoport JL (1997) A development functional MRI study of prefrontal activation during performance of a go-no go task. J Cogn Neurosci 9:835-847.

Chudasama Y, Passetti F, Rhodes SEV, Lopian D, Desai A, Robbins TW (2003) Dissociable aspects of performance on the 5 choice serial reaction time task following lesions of the dorsal anterior cingulate, infralimbic, and orbitofrontal cortex in the rat: differential effects on selectivity, impulsivity, and compulsivity. Behav Brain Res, in press.

Dias R, Robbins TW, Roberts AC (1996) Dissociation in prefrontal cortex of affective and attentional shifts. Nature 380:69-72.

Dickinson A, Balleine B (1994) Motivational control of goal-directed action. Anim Learn Behav 22:1-18.

Eichenbaum H, Clegg RA, Feeley A (1983) Reexamination of functional subdivisions of the rodent prefrontal cortex. Exp Neurol 79:434-451.

Floyd NS, Price JL, Ferry AT, Keay KA, Bandler R (2001) Orbitomedial prefrontal cortical projections to hypothalamus in the rat. J Comp Neurol 432:307-328. 
Fuster J (1989) The prefrontal cortex: anatomy, physiology and neuropsychology of the frontal lobe, Ed 2. New York: Raven.

Granon S, Poucet B (1995) Medial prefrontal lesions in the rat and spatial navigation: evidence for impaired planning. Behav Neurosci 109:474-484.

Hurley KM, Herbert H, Moga MM, Saper CB (1991) Efferent projections of the infralimbic cortex of the rat. J Comp Neurol 308:249-276.

Iversen SD, Mishkin M (1970) Perseverative interference in monkeys following selective lesions of the inferior prefrontal convexity. Exp Brain Res 11:376-386.

Jones B, Mishkin M (1972) Limbic lesions and the problem of stimulusreinforcement associations. Exp Neurol 36:362-377.

Kolb B, Nonneman AJ, Singh RK (1974) Double dissociation of spatial impairments and perseveration following selective prefrontal lesions in rats. J Comp Physiol Psychol 87:772-780.

Mackintosh NJ (1983) Conditioning and associative learning. Oxford: Clarendon.

Macmillan NA, Creelman CD (1991) Detection theory: a user's guide. Cambridge, UK: Cambridge UP.

Miller EK (2000) The prefrontal cortex and cognitive control. Nat Rev Neurosci 1:59-65.

Mishkin M (1964) Perseveration of central sets after frontal lesions in man. In: The frontal granular cortex and behaviour (Warren JM, Akert K, eds), pp 313-334. New York: McGraw-Hill.

Neafsey EJ (1990) Prefrontal cortical control of the nervous system: anatomical and physiological observations. Prog Brain Res 85:147-166.

O’Doherty J, Kringelbach ML, Rolls RT, Hornak J, Andrews C (2001) Abstract reward and punishment representations in the human orbitofrontal cortex. Nat Neurosci 4:95-102.

Öngür D, Price JL (2000) The organization of networks within the orbital and medial prefrontal cortex of rats, monkeys and humans. Cereb Cortex 10:206-219.

Parkinson JA, Robbins TW, Everitt BJ (2000a) Dissociable roles of the central and basolateral amygdala in appetitive emotional learning. Eur J Neurosci 12:405-413.

Parkinson JA, Willoughby PJ, Robbins TW, Everitt BJ (2000b) Disconnection of the anterior cingulate cortex and nucleus accumbens core impairs Pavlovian approach behavior: further evidence for limbic cortical-ventral striatopallidal systems. Behav Neurosci 114:42-63.

Passetti F, Chudasama Y, Robbins TW (2002) The frontal cortex of the rat and visual attentional performance: dissociable functions of distinct medial prefrontal subregions. Cereb Cortex 12:1254-1268.
Paxinos G, Watson C (1997) The rat brain in stereotaxic coordinates, Ed 2. Sydney: Academic.

Phillipson OT, Griffiths AC (1985) The topographic order of inputs to the nucleus accumbens in the rat. Neuroscience 16:275-296.

Ragozzino ME, Detrick S, Kesner RP (1999) Involvement of the prelimbicinfralimbic areas of the rodent prefrontal cortex in behavioral flexibility for place and response learning. J Neurosci 19:4585-4594.

Rahman S, Sahakian BJ, Hodges JR, Rogers RD, Robbins TW (1999) Specific cognitive deficits in mild frontal variant frontotemporal dementia. Brain 122:1469-1493.

Rogers RD, Owen AD, Middleton HC, Williams EJ, Pickard JD, Sahakian BJ, Robbins TW (1999) Choosing between small, likely rewards and large, unlikely rewards activates inferior and orbital prefrontal cortex. J Neurosci 20:9029-9038.

Rolls ET (1996) The orbitofrontal cortex. Philos Trans R Soc Lond B Biol Sci 351:1433-1444.

Rolls ET (2000) The orbitofrontal cortex and reward. Cereb Cortex 10:284-294.

Rolls ET, Hornak J, Wade D, McGrath J (1994) Emotion-related learning in patients with social and emotional changes associated with frontal damage. J Neurol Neurosurg Psychiatry 57:1518-1524.

Schoenbaum G, Chiba AA, Gallagher M (2000) Rapid changes in functional connectivity in orbitofrontal cortex and basolateral amygdala during learning and reversal. J Neurosci 20:5179-5189.

Schoenbaum G, Nugent S, Saddoris MP, Setlow B (2002) Orbitofrontal lesions in rats impair reversal but not acquisition of go, no-go odor discriminations. NeuroReport 13:885-890.

Seamans JK, Floresco SB, Phillips AG (1995) Functional differences between the prelimbic and anterior cingulate regions of the rat prefrontal cortex. Behav Neurosci 109:1063-1073.

Sutherland NS, Mackintosh NJ (1971) Mechanisms of animal discrimination learning. New York: Academic.

Tremblay L, Schultz W (1999) Relative reward preference in primate orbitofrontal cortex. Nature 398:704-708.

Volkow ND, Fowler JS (2000) Addiction, a disease of compulsion and drive: involvement of the orbitofrontal cortex. Cereb Cortex 10:318-325.

Williams DR, Williams H (1969) Auto-maintenance in the pigeon: sustained pecking despite contingent nonreinforcement. J Exp Anal Behav 12:511-520.

Winer BJ (1971) Statistical principles in experimental design, Ed 2. New York: McGraw-Hill. 